\title{
Temperature dependence of the current in Schottky-barrier source-gated transistors
}

\author{
R. A. Sporea, M. Overy, J. M. Shannon, and S. R. P. Silva \\ Advanced Technology Institute, University of Surrey, Guildford, GU2 7XH, Surrey, United Kingdom
}

The temperature dependence of the drain current is an important parameter in thin-film transistors. In this paper, we propose that in source-gated transistors (SGTs), this temperature dependence can be controlled and tuned by varying the length of the source electrode. SGTs comprise a reverse biased potential barrier at the source which controls the current. As a result, a large activation energy for the drain current may be present which, although useful in specific temperature sensing applications, is in general deleterious in many circuit functions. With support from numerical simulations with Silvaco Atlas, we describe how increasing the length of the source electrode can be used to reduce the activation energy of SGT drain current, while maintaining the defining characteristics of SGTs: low saturation voltage, high output impedance in saturation and tolerance to geometry variations. In this study we apply the dual current injection modes to obtain drain currents with high and low activation energies and propose mechanisms for their exploitation in future large-area integrated circuit designs.

\section{INTRODUCTION}

\section{A. Schottky barrier source-gated transistors (SB-SGTs)}

Source-gated transistors (SGTs) ${ }^{1-3}$ are formed by deliberately engineering a potential barrier at the source of a staggeredelectrode thin-film field effect transistor (TFT). The depletion region which forms at the source barrier extends across the semiconductor layer when a small (usually below 2V) reverse bias is applied to the drain, leading to source pinch-off and drain current saturation at significantly lower drain voltage in comparison to a conventional TFT. Figure 1a shows a schematic cross-section of a $n$-type SGT under positive gate and drain bias.

The source barrier is reverse biased, and as the reverse bias increases, the depletion envelope widens until all the negative charge at the drain end of the source is depleted. In this condition, the drain current is saturated, as any further increase in $V_{D}$ is dropped across the depleted region and has little effect on the field and voltage distribution at the source barrier ${ }^{2,4}$. Regardless of the current injection mechanism at the source ${ }^{3,5,6}$, the intentional use of a source barrier in an otherwise conventional TFT structure leads to major differences in the transistor (output) characteristic: low-voltage saturation 2, 3, 7-10, flat saturated output characteristics ${ }^{3,7,9,11}$, tolerance to geometrical variations ${ }^{4,12}$, bias stress stability ${ }^{13}$ and improved 
current levels in low mobility materials ${ }^{14}$. Both digital ${ }^{15}$ and analog applications ${ }^{14,}$ 16-19 could benefit from using these devices, in terms of amplification, energy efficiency, uniformity of electrical performance, and reliability. Figure $1 \mathrm{~b}$ and c show, respectively, output and transfer characteristics measured on an n-type polysilicon SGT with Schottky source barrier. Note the low saturation voltage, flat characteristics and exponential dependence of drain current on gate voltage above threshold and in saturation.

\section{B. Temperature behavior of drain current in FETs and SGTs}

The field and voltage distributions in a SGT are two-dimensional, and it is therefore difficult to find a simple analytical expression for the current for all geometries. A lumped circuit model is shown in Figure 1d, but the value of each component can only be found using 2-D computer analysis. Essentially, there are two current sources, with different characteristics. $I_{l}$ is the current crossing the barrier in the high field (depleted) region at the drain end of the source; $I_{2}$ is the current crossing the source barrier in the low field region (bulk) of the source contact. A complication occurs because the low field current, $I_{2}$, can be restricted by the fully depleted region at the drain end of the source. This effect is the same as the action of a junction field-effect transistor (JFET).

The drain current is therefore determined by two mechanisms, and which one dominates is determined by device geometry, the source barrier height, and the gate biasing condition. For low barriers and long sources we expect the current to be restricted by the JFET action and by the resistive path through the semiconductor in the bulk region of the source. For high barriers and short sources, the current should be determined by the field dependence of the barrier at the end of the source ${ }^{5}$. All of these mechanisms are temperature activated, because there is a barrier, either at the source contact or at the JFET, which determines current flow, and we expect the general Arrhenius expression to be relevant:

$$
I_{D} \propto \exp \left(-\frac{q \phi_{B}}{k T}\right)
$$

where $\phi_{\mathrm{B}}$ is the effective barrier for carrier transport.

For a Schottky barrier source, the field dependence of the barrier height is determined by image force lowering and quantum mechanical tunneling through the barrier ${ }^{20}$. It has been shown that when field $E_{S}$ is high, the barrier lowering can be approximated by:

$$
\Delta \phi_{B} \approx \alpha E_{s}
$$

where $\alpha$ is an effective barrier lowering constant. A general equation for barrier lowering is:

$$
\Delta \phi_{B} \approx \alpha E^{\gamma}+\beta \frac{q}{4 \pi \varepsilon_{S}} E^{0.5},
$$


where the first term is more accurate at high fields, and the second, more accurate at low fields.

From (1), the activation energy $\left(E_{A}\right)$ for current transport is

$$
E_{A}=-\frac{\partial(\ln I)}{\partial(1 / k T)}
$$

where $I$ is the current, $k$ is Boltzmann's constant and $T$ is temperature.

Determination of $E_{A}$ enables us to estimate the temperature coefficient of the drain current (TC) and its dependence on source length. Clearly, a low $E_{A}$ leads to low temperature dependence. As a guide, for a $30 \mathrm{~K}$ increase above room temperature, $\Delta E_{A}$ $=30 \mathrm{meV}$ represents a doubling of current, and an order of magnitude increase has an associated $\Delta E_{A}$ of approximately $64 \mathrm{meV}$.

SGTs with high Schottky source barriers tend to have large positive TC. Crystalline MOSFETs have a small negative TC resulting from the increased phonon scattering at high temperature with an associated reduction in carrier mobility ${ }^{21}$. In a disordered-semiconductor thin film field effect transistor, this behavior is usually overcompensated by the change of carrier trapping with temperature, leading to a small, positive $\mathrm{TC}^{21}$. A large positive $\mathrm{TC}$ can be detrimental to circuit operation, first through the positive feedback potentially leading to catastrophic failure which might result as temperature is increased, and second in the unfavorable changes in electrical characteristics, which could necessitate compensation circuitry, complex designs and larger design margins. In this study, we investigate means of controlling the TC of SGT drain current through leveraging of device-specific physics in their design.

\section{SIMULATION SETUP}

The structure was defined in Silvaco Atlas as a two-dimensional cross-section. For consistency with previous studies ${ }^{2-6}$, 9, 22 we analyzed an n-type amorphous silicon device with default atlas material parameters and the following properties: ohmic drain contact; nominal source barrier height set by choosing the work function of the metal to be higher by $\phi_{\mathrm{B} 0}=0.5 \mathrm{eV}$ than the semiconductor electron affinity (actual value will be affected by interface properties); source-drain gap $d=5 \mu \mathrm{m}$; device width $W=1 \mu \mathrm{m}$; eight exponentially spaced values for source length $S: 0.2,0.5,1.2,2.9,7,17,41,100 \mu \mathrm{m}$; semiconductor thickness $t_{S}=100 \mathrm{~nm}$; insulator $\left(\mathrm{SiO}_{2}\right)$ thickness $t_{i}=100 \mathrm{~nm}$.

We are investigating the effect of source length and almost all the source is subject to a low field because most of the drain potential is dropped across the pinch-off region at the end of the source (Figure 1). For this reason, the simulation 
parameters in Equation (3) were set to $\alpha=0$ and $\beta=1$, and therefore confined to image force lowering, which is dominant at low fields.

In order to achieve good resolution in the important area around the edge of the source closest to the drain, meshing in the $x$ direction was kept constant at $5 \mathrm{~nm}$ at that point, and allowed to vary as a proportion of $S$ at the other edge. The ambient temperature range considered was $300-360 \mathrm{~K}$.

\section{RESULTS AND DISCUSSION}

\section{A. Modes of operation and source geometry}

As outlined in Section I B, there are two distinct modes of operation of the SGT. The first mechanism is specific to the area of the source in which pinch-off occurs in the semiconductor (Figure 1a) and has a high dependence on electric field (from gate or drain). As the semiconductor is depleted in this region (first few tens to hundreds of nanometers from the edge of the source closest to the drain inwards), the electric field can penetrate to the metal-semiconductor contact and contribute to barrier lowering from Schottky effect and tunnelling. Thus, at high electric field, the barrier in this region of the source can be modulated, and since the reverse current of the contact is exponentially dependent on effective barrier height ${ }^{20}$, large changes in current $\left(I_{1}\right)$ can be effected by changing the potential on the gate. The drain bias, however, has a similar effect on this area of the source, so without purposeful screening of the source edge from drain electric field ${ }^{11}$, output characteristics have a drain voltage dependence of the current in saturation ${ }^{5,9}$.

The second mechanism involves the whole area of the source (for the benefit of visualization we will call this the "bulk" of the source, as opposed to the "edge" referred to above). The current here $\left(I_{2}\right.$ in Figure 1d) is controlled by the combination of a distributed resistive network in the semiconductor under the source and the JFET-like region at the semiconductor-insulator interface where pinch-off occurs $\left(D_{p}\right)$. The resistance in the $x$ direction is represented by the accumulation layer created by the applied gate bias at the semiconductor-insulator interface. In the $y$ direction, the resistance is given by the average resistivity of the semiconductor and its thickness. (Figure 1d $)^{3,9}$ The reverse biased source barrier $\left(D_{s}\right.$ in Figure 1d) plays a role in current control, not as a current-limiting mechanism, but rather as a non-linear potential drop. Simply, the second mechanism is a two-dimensional potential gradient problem, in which (for $V_{G}$ above "threshold") $V_{S A T 1}=\left(V_{G}-V_{T}\right) \cdot C_{i} \cdot\left(C_{i} /\right.$ $C_{S}$ ) is applied to the anode of $D_{p}$ ("floating source" of the parasitic FET which forms between this point and the ohmic drain) in Figure $1 d^{3,23}$. 
Figure 1e shows the current density $J_{S}$ crossing the source-semiconductor interface for different source lengths. The total drain current will be the integral of $J_{S}$ across the length of the source. The biasing condition considered $\left(V_{G}, V_{D}\right)$ ensures that the device is operating in saturation and that the current is controlled by the source. The plot shows the current density injected at that point of the source vs. position along the source, starting at the edge closest to the drain. Five curves are presented, for devices with source lengths of $1,3,5,10$ and $100 \mu \mathrm{m}$, respectively. In the first $\sim 200 \mathrm{~nm}$ of the source, current injection has a very similar profile, regardless of $S$. This is the region where the first injection mechanism $\left(I_{l}\right)$ dominates. Further along the source length, the curves diverge when $S$ changes.

As an example, consider the curves for $S=1$ and $10 \mu \mathrm{m}$. The current injected at $x=1 \mu \mathrm{m}$ by the shorter source is larger. This is due to the potential drop produced by current flowing in the accumulation layer ( $R_{c h}$ chain) (Figure $1 \mathrm{~d}$ ). In the $S=10 \mu \mathrm{m}$ device, the total current injected between $x=10 \mu \mathrm{m}$ and $x=1 \mu \mathrm{m}$ flowing between $x=1 \mu \mathrm{m}$ and $x=0$ creates a potential drop at $x=1 \mu \mathrm{m}$ which does not exist for the $S=1 \mu \mathrm{m}$ device. The total current is higher in the longer-source device owing to the larger injection area.

Following the analysis above, we can see that increasing $S$ further leads to a condition where the potential in the channel at the far end of the source reaches a value for which no voltage is dropped across the respective Rsc and no current is injected by that region of the source (Figure 1e). Increasing $S$ beyond this value $\left(S_{S A T}\right)$ does not lead to an increase in total current. The value of $S_{S A T}$ depends on the resistivity of the semiconductor channel (magnitude of $R_{c h}$ ), which in turn is modulated by applied gate bias. Figure 2a shows the change in $S_{S A T}$ with $V_{G}$ and the saturation of the current with increasing $S$ above $S_{S A T}$.

\section{B. Source length and its influence on mode of operation}

Changes in device operation are observed, depending on which of the two current injection mechanisms dominates. The transconductance of the drain current $\left(g_{m}\right.$, Figure 2 b) shows a definite kink when switching between modes of operation ${ }^{5}$. A longer $S$ allows more current to be injected from the bulk of the source, pushing the gate voltage at which the kink occurs to higher values. This is due to the fact that more barrier lowering needs to occur for longer $S$ in order for current $I_{1}$ (Figure 1d) to increase enough to compete and overtake $I_{2}$. For a given $S$, at $V_{G}$ below the kink in $g_{m} I_{2}$ dominates, while above, $I_{1}$ dominates.

\section{Activation energy of drain current}

\section{Source length and activation energy}


The observations above and in Ref. 5 have significant implications on the temperature dependence of drain current. We plot the activation energy $\left(E_{A}\right)$ of $I_{D}$ vs. gate bias for different values of $S$. Neglecting the low-voltage, sub-threshold range of $V_{G}$, a large range of $E_{A}$ is observed. The nominal value for the barrier height (metal work function minus semiconductor electron affinity) in the model was $\phi_{\mathrm{B} 0}=0.5 \mathrm{eV}$, but the effective barrier $\left(\phi_{\mathrm{B} 0}\right)$ will depend on surface characteristics and is expected to be higher. Importantly, $E_{A}$ decreases with $S$ and for the largest $S$ activation energy is $\sim 0.3 \mathrm{eV}$, significantly smaller than $\phi_{\mathrm{B} 0}$. Shorter values of $S$ produce curves which initially dip substantially below $\phi_{\mathrm{B} 0}$ ' but later increase back towards $\phi_{\mathrm{B} 0}$ at high $V_{G}$. The deviation from the curve of minimum $E_{A}$ happens for different values of $S$ at the same $V_{G}$ as the kink in transconductance, since the change in mode of operation (and current control mechanism) brings about a change in thermal properties of the injected current. For very short $S$, the current has a high $E_{A}$ which decreases somewhat with $V_{G}$, according to the field-dependent barrier lowering model enabled in the simulator $(\beta=1)$.

In brief, Figure 2c shows that depending on the dominant injection mechanism, the current can have significantly different activation energy. The envelope at higher $E_{A}$ is the activation energy for $I_{1}$ while the lower envelope is for $I_{2}$. Current $I_{l}$, dominant for short sources or very high $V_{G}$ has, as expected, an activation energy close to the effective barrier height $\phi_{\mathrm{B} 0}$, which is pulled down as $V_{G}$ increases. Current $I_{2}$, conversely, has a very low activation energy which cannot be explained by the properties of the barrier and must be imparted by the current restriction mechanisms of $I_{2}$.

\section{Effects of other parameters on activation energy}

Figure 3 shows the activation energy of drain current vs. $V_{G}$ for SGTs with extreme values of source length: 0.2 and $100 \mu \mathrm{m}$, for three values of nominal barrier height $\phi_{\mathrm{B} 0}$, in the absence of field-induced barrier lowering $(\beta=0)$. For the short source device, in which $I_{1}$ dominates and is controlled exclusively by the barrier, $E_{A}$ correlates with $\phi_{\mathrm{B} 0}$. With no field-dependent barrier lowering, $E_{A}$ does not change with $V_{G}$ above threshold. Increases in $\phi_{\mathrm{B} 0}$ translate in equal increases of $E_{A}$. For the long source $\left(I_{2}\right.$ dominates), smaller increases in $E_{A}$ are seen than the respective increases of $\phi_{\mathrm{B} 0}$. We explain this as a consequence of current control in this mode of operation: an increase $\phi_{\mathrm{B} 0}$ reduces the voltage drop available across the semiconductor; with little change to the resistance $R_{s c}$, the current injected from a point in the bulk of the source is less for higher $\phi_{\mathrm{B} 0}$, following the reduction in potential difference between the two sides of the semiconductor. In this mode of operation, there is also significant modulation of $E_{A}$ with $V_{G}$, which will be discussed in the next section.

In Figure 3, the lack of field-induced barrier lowering is unrealistic. Supplementary Figure SF $1 \mathrm{a}^{24}$ shows the effect of enabling field-dependent barrier lowering $(\beta=1)$. A FET with the same geometry and characteristics was simulated for 
comparison. Its drain current activation energy (Supplementary Figure SF1b ${ }^{24}$ ) the small but positive, consistent with current understanding of these devices ${ }^{21}$.

\section{Split source didactical model}

In order to be able to graphically show the effect of $I_{1}$ and $I_{2}$, the following structure was studied: the source of the device in Figure $1 \mathrm{~d}$ was split into two electrodes $\left(S_{1}=0.2 \mu \mathrm{m}\right.$ and $\left.S_{2}=95 \mu \mathrm{m}\right)$ separated by a $4.8 \mu \mathrm{m}$ gap (Figure $\left.4 \mathrm{a}\right)$. In the first approximation, $S_{1}$ is injecting $I_{1}$ and $S_{2}$ is injecting $I_{2}$. The functional difference between this structure and the original is the gap between $S_{1}$ and $S_{2}$ which introduces a $R_{c h}$ component which was not present in Figure 1d, potentially lowering the magnitude of $I_{2}$ somewhat by the discussed potential drop. However, this effect can be discounted in the present qualitative discussion. More importantly, the edge of $S_{2}$ closest to $S_{1}$ does not induce pinch-off during operation, so there is no $I_{1}$-type component originating form $S_{2}$, and the $I_{2}$-type component injected by $S_{1}$ is comparatively small, given its size.

While this type of structure is impractical to fabricate with current technology, the simulation allows us to identify and understand contributions from the edge and the bulk of a continuous source electrode in a practical device.

The transfer characteristic in Figure $4 \mathrm{~b}$ shows the contribution to drain current of the currents injected by $S_{1}$ and $S_{2}$ (again, for practical purposes equivalent to $I_{1}$ and $I_{2}$ ). $S_{1}$ and $S_{2}$ are both at $0 \mathrm{~V}$ potential. The drain current is the sum $I_{1}$ and $I_{2}$. At very low gate bias, it is made up exclusively of $I_{1}$. With increasing $V_{G}, I_{1}$ becomes limited by the reverse saturation current of the contact at $S_{1}$ (for simplicity, no barrier lowering was considered in this theoretical study; $\beta=0$ ). At the same time, $S_{2}$ increases as the accumulation layer becomes more conductive and surpasses $I_{1}$ (at a voltage where the kink in the $g_{m}$ characteristic occurs, see Figure 2b), eventually becoming the dominant component of $I_{D}$.

In Figure 4c, output-like characteristics are drawn for $I_{1}, I_{2}$ and $I_{D}$, for different values of $V_{G}$. We notice the low modulation of $I_{1}$ and its low magnitude at high $V_{G} . I_{D}$ is exclusively composed of $I_{2}$ at high gate bias. The change in saturation voltage $V_{S A T 1}$ with $V_{G}$ is, to a good approximation, only controlled by the electrostatics of the semiconductor-insulator stack and remains unchanged regardless of the proportion of $I_{1}$ and $I_{2}$.

Supplementary Figure SF2 ${ }^{24}$ illustrates the composition of $I_{D}$ in terms of $I_{1}$ and $I_{2}$ at different gate biases, showing graphically that $I_{D}$ has only slight dependence on $I_{1}$, particularly at high $V_{G}$, and that is almost entirely made up of $I_{2}$, apart from the very low current region at low $V_{G}$.

Given that $I_{1}$ and $I_{2}$ have very different activation energies, the temperature coefficient of the drain current will be defined by the dominant of the two. Figure 5a shows the activation energy vs. gate voltage plot for drain current in a device with 
constant $\phi_{\mathrm{B} 0}\left(S_{1}\right)=0.45 \mathrm{eV}$ and varying $\phi_{\mathrm{B} 0}\left(S_{2}\right)$ from 0.2 to $0.55 \mathrm{eV}$. When $\phi_{\mathrm{B} 0}\left(S_{2}\right)$ is high, $I_{2}$ contribution is low and $E_{A}$ is given by $S_{1}$. As $\phi_{\mathrm{B} 0}\left(S_{2}\right)$ decreases, $I_{2}$ becomes higher and dominates in the composition of $I_{D}$. This is a similar effect to that obtained by increasing $S$ in the original structure. The activation energy of $I_{1}$ and $I_{2}$ are plotted together with that of $I_{D}$ in Figure $5 \mathrm{~b}$. We see how drain current $E_{A}$ switches between the two as $I_{2}$ increases with $V_{G}$. The composition of drain current is shown graphically in the cross-section in Figure 5c, obtained by probing across the device $2.5 \mathrm{~nm}$ into the semiconductor at the insulator interface. Current builds up along the length of $S_{2}$ and it plateaus as it crosses the gap between $S_{2}$ and $S_{1}$. The contribution of $S_{2}$ is added on to form the total current which passes through the accumulated channel and is collected at the drain. The relative magnitude of $I_{1}$ and $I_{2}$ dictate the dominant mode of operation, and also the activation energy of $I_{D}$.

\section{Implications for device design}

The easiest way of ensuring dominance of $I_{2}$ over $I_{1}$ is through sizing of the source electrode. Longer $S$ shifts the ratio of $I_{1}$ and $I_{2}$ in the composition of drain current towards the latter. Thus, the activation energy of drain current tends to that of $I_{2}$, which is lower. Extremely long values of $S$ have no effect, as the current tends to saturate with $S$ due to resistive effects in the semiconductor (Figure 1d).

For the geometries and biasing conditions considered, which originate in practical devices studied extensively in the past, we found that values of source length, $S$, in the $30-50 \mu \mathrm{m}$ range are best suited for obtaining low temperature coefficient of drain current. From a fabrication point of view, such $S$ is not wasteful of layout area, considering the conventional sizing of (doped) contact region and design rules for contact metallization. Furthermore, source-drain gap can be made as small as the technology will allow in SGTs while maintaining flat saturated characteristics, saving area in the same direction as $S$.

Relatively long $S$ is preferred for a related reason: $I_{1}$ is electric field-dependent; $I_{2}$ is less so. When $I_{2}$ dominates, the flatness of the output characteristics is superior without the need for a specially-designed field relief structure ${ }^{5,11}$. Long source devices should have improved intrinsic gain characteristics.

Saturation voltage, $V_{S A T 1}{ }^{7}$ remains low compared to conventional FETs, even when $S$ is large. A slight increase in $V_{S A T I}$ may be observed for long $S$, as the current below saturation follows the FET linear region envelope (longer $S$ entails total current injected at the source, which is closer to the FET channel conductance envelope for that particular gate and drain bias combination).

\section{E. Implications for circuit design}


The gate overlaps the whole area of the source. The capacitance introduced by large $S$ (large source-gate overlap) may indeed lower the operating frequency of such devices. However, we expect the utility of SGTs to be in localized areas of circuits such as bias and signal generation, initial signal amplification, active loads to high gain stages, where their other characteristics will play a major role and where speed is not the principal requirement.

Source-drain gap is inconsequential in SGTs over a large range. With changes in $S$, however, come (modest) changes in current and temperature coefficient. Longer values of $S$, then, ensure that processing variations resulting from poor registration or thermal expansion/shrinkage of the substrate during fabrication are easily tolerated. First, the total drain current changes very little with changes in $S$ around a certain value $S_{S A T}$. Second, as a proportion of total $S$, variations in the micron range are comparatively small. Together these result in superior matching of electrical characteristics of devices in different parts of the substrate, and improved consistency across substrates and fabrication runs.

A similar benefit in terms of uniformity of performance across a large area arises from the lower temperature coefficient of the drain current for devices with longer $S$. Different parts of the chip are prone to temporal and areal variations in temperature during operation. Temperature dependence is minimized when $S$ is long, permitting more relaxed design margins, simpler or no temperature compensation and improved operating temperature window.

Lastly, the low increase in effective saturation voltage, $V_{S A T I}$ with $S$ suggests that longer-source SGTs are still capable of being used in power-efficient designs, with low series voltage drop, meaning lower power supply levels or, conversely, the ability to design with additional cascaded devices between the rails, such as in cascade architectures.

\section{CONCLUSION}

Thin-film source-gated transistors are field-effect devices with construction very similar to conventional transistors. A source barrier deliberately engineered at the source radically changes the operation of such devices, allowing them to achieve low saturation voltage and high output impedance in saturation, along with stability under bias stress and tolerance to geometrical variations. Numerous analog as well as digital applications can benefit from the energy efficiency and gain characteristics of such devices.

Two mechanisms for current injection at the source have been identified. Firstly, the first few hundreds of nm of the source from the edge closest to the drain operate in a high electric field, high activation energy, and high barrier modulation regime. The temperature coefficient of this current $\left(I_{1}\right)$ is comparatively high, unless substantial barrier lowering occurs under electric field. Secondly, the remaining area (bulk) of the source $\left(I_{2}\right)$ injects current in a low electric field, low activation energy, low 
source (metal/semiconductor) barrier modulation regime, which is controlled by a resistive network in the source region of the semiconductor, along with a JFET-like component in the pinch-off region at the edge of the source closest to the drain, on the semiconductor-insulator interface. $I_{2}$ has a low temperature coefficient.

In this paper we have described how the activation energy of total drain current depends on the activation energies of $I_{1}$ and $I_{2}$, but also, importantly, of the ratio of $I_{1}$ and $I_{2}$. In order to reduce the temperature coefficient of drain current, the lowactivation energy $I_{2}$ is required to be significantly greater than $I_{l}$. This way, drain current activation energy approaches that of $I_{2}$.

Notably, lowering the activation energy of drain current by a simple method such as increasing $S$ will simplify design of SGT-containing circuit blocks in a large-area system where spatial temperature variations are likely to occur, reducing the need for compensation circuitry. At the values of $S$ needed, process variations of $S$ and $d$ are well tolerated, with no practical changes in electrical characteristic.

The simple structure and design parameters of SGTs, in conjunction with its operating characteristics recommend these devices as powerful circuit design tools for future energy-efficient, high performance large area electronic systems.

\section{ACKNOWLEDGMENTS}

The work of R. A. Sporea is supported by the Royal Academy of Engineering Academic Research Fellowship Program.

\section{REFERENCES}

1. S. D. Brotherton, Introduction to Thin Film Transistors: Physics and Technology of TFTs. (Springer, 2013).

2. J. M. Shannon and E. G. Gerstner, Electron Device Letters, IEEE 24 (6), 405-407 (2003).

3. A. Valletta, L. Mariucci, M. Rapisarda and G. Fortunato, Journal of Applied Physics 114 (6), 064501 (2013).

4. J. M. Shannon and E. G. Gerstner, Solid-State Electronics 48 (7), 1155-1161 (2004).

5. J. M. Shannon, R. A. Sporea, S. Georgakopoulos, M. Shkunov and S. R. P. Silva, Electron Devices, IEEE Transactions on 60 (8), 2444-2449 (2013).

6. T. Lindner, G. Paasch and S. Scheinert, Electron Devices, IEEE Transactions on 52 (1), 47-55 (2005).

7. R. A. Sporea, M. J. Trainor, N. D. Young, J. M. Shannon and S. R. P. Silva, Electron Devices, IEEE Transactions on 57 (10), 2434-2439 (2010).

8. R. A. Sporea, M. J. Trainor, N. D. Young, X. Guo, J. M. Shannon and S. R. P. Silva, Solid-State Electronics 65-66 (0), 246-249 (2011). 
9. A. Valletta, A. Daami, M. Benwadih, R. Coppard, G. Fortunato, M. Rapisarda, F. Torricelli and L. Mariucci, Applied Physics Letters 99 (23), 233309 (2011).

10. A. M. Ma, M. Gupta, F. R. Chowdhury, M. Shen, K. Bothe, K. Shankar, Y. Tsui and D. W. Barlage, Solid-State Electronics 76 (0), 104-108 (2012).

11. R. A. Sporea, M. J. Trainor, N. D. Young, J. M. Shannon and S. R. P. Silva, Electron Devices, IEEE Transactions on 59 (8), 2180-2186 (2012).

12. R. A. Sporea, G. Xiaojun, J. M. Shannon and S. R. P. Silva, presented at the Semiconductor Conference, 2009. CAS 2009. International, 2009 (unpublished).

13. J. M. Shannon, Applied Physics Letters 85 (2), 326-328 (2004).

14. G. Xiaojun, F. Balon, R. A. Hatton and J. M. Shannon, presented at the Flexible Electronics and Displays Conference and Exhibition, 2008, 2008 (unpublished).

15. R. A. Sporea, M. J. Trainor, N. D. Young, J. M. Shannon and S. R. P. Silva, Sci. Rep. 4 (2014).

16. X. Xiaoli, F. Linrun, H. Shasha, J. Yizheng and G. Xiaojun, Electron Device Letters, IEEE 33 (10), 1420-1422 (2012).

17. R. A. Sporea, X. Guo, J. M. Shannon and S. R. P. Silva, in Thin Film Transistors 10, edited by Y. Kuo (2010), Vol. 33, pp. 419-424.

18. R. A. Sporea, S. Georgakopoulos, X. Xu, X. Guo, M. Shkunov, J. M. Shannon and S. R. P. Silva, MRS Online Proceedings Library 1553, null-null (2013).

19. R. A. Sporea, J. M. Shannon and S. R. P. Silva, presented at the Device Research Conference (DRC), 201169 th Annual, 2011 (unpublished).

20. S. M. Sze and K. K. Ng, Physics of Semiconductor Devices, 3rd Edition, Third Edition ed. (Wiley and Sons, 2006).

21. H. Ibach and H. Lüth, Solid-State Physics: An Introduction to Principles of Materials Science, Fourth edition ed. (Springer, 2009).

22. F. Balon, J. M. Shannon and B. J. Sealy, Applied Physics Letters 86 (7), 073503 (2005).

23. J. M. Shannon and F. Balon, Electron Devices, IEEE Transactions on 56 (10), 2354-2356 (2009).

24. See supplemental material at ... for further discussion of activation energy dependence on constructive parameters and for relative contributions of current injected by electrodes $S_{1}$ and $S_{2}$ to drain current. 


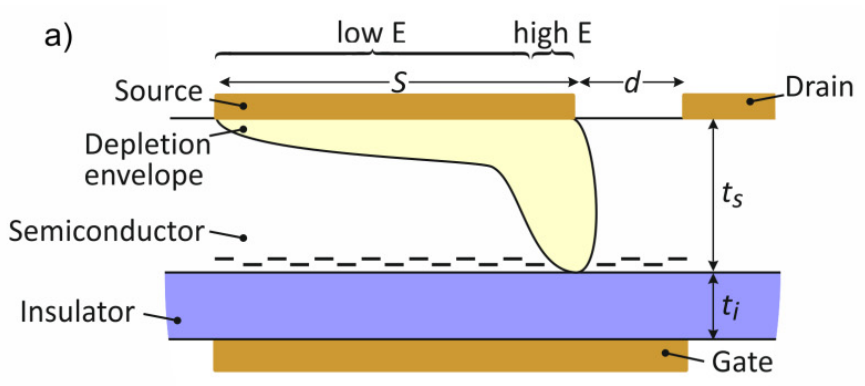

d)
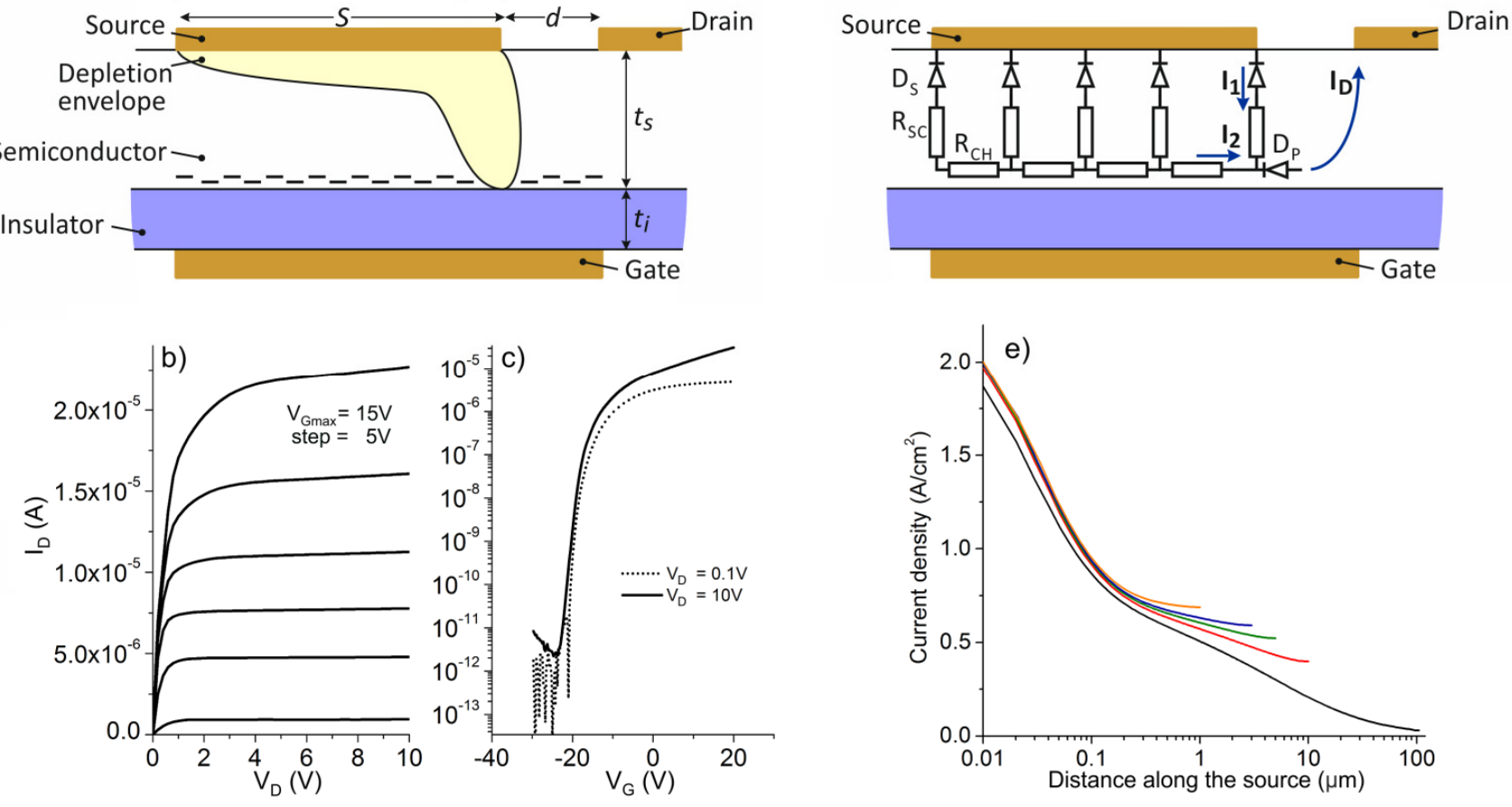

FIG. 1. a) Schematic cross-section of an n-type bottom gate source-gated transistor (SGT) under positive gate and drain biases, showing the envelope of the depletion region under the source; measured b) output and c) transfer characteristics of a polysilicon $n$-type SGT, fabricated as described in ${ }^{7}$; d) The same cross-section as a) showing the distribution of current restriction elements within the semiconductor and at the source metal-semiconductor contact, along with the two distinct components of drain current $\left(\mathrm{after}^{3}\right)$; e) simulated current density crossing the source-semiconductor interface at every distance along the source contact away from the edge closest to the drain, illustrating the decreasing current density with distance for long sources, as a result of the resistive network shown in d). 

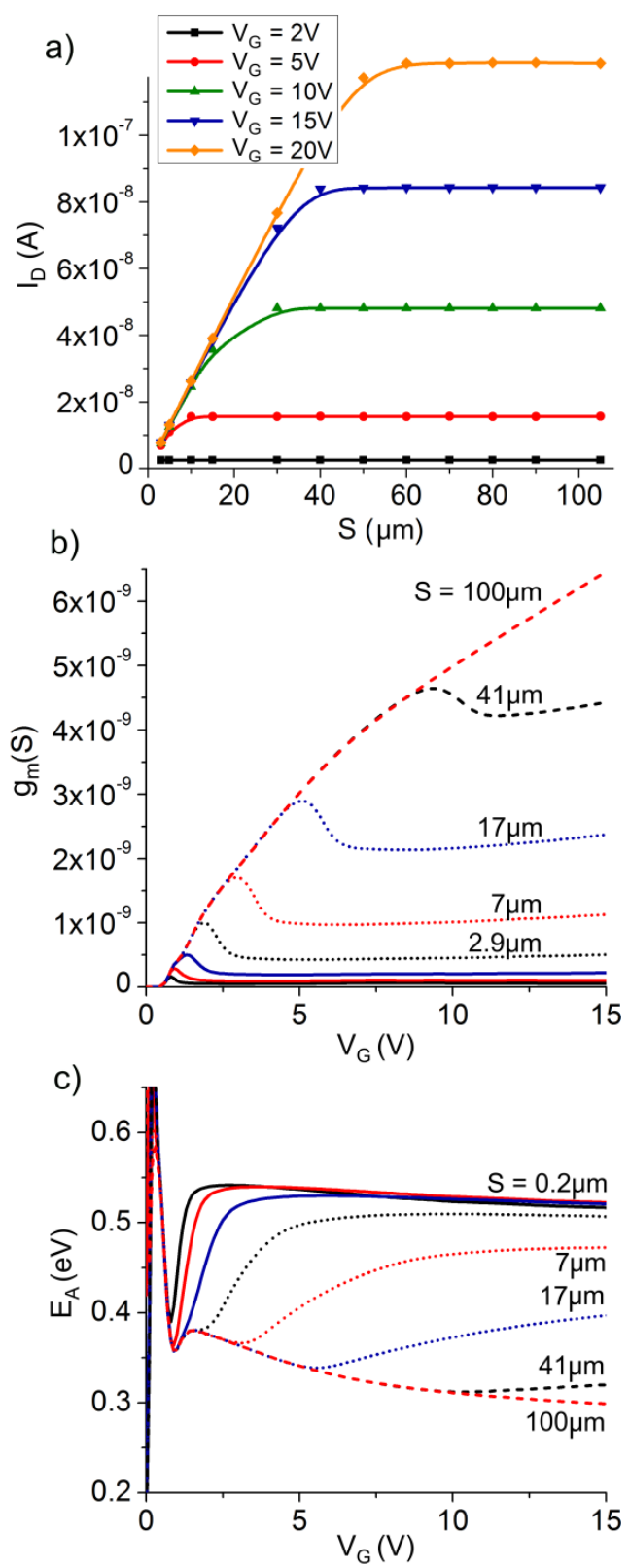

FIG. 2. Simulation of SGT electrical behavior with changes in source length. a) Dependence of saturated drain current on gate bias and source electrode length, $S ;$ b) Transconductance, $g_{m}$ shows a kink in its gate voltage dependence. The kink occurs at higher voltages for longer $S$ and is due to the change of dominant current injection mechanism. Bottom to top $S=0.2,0.5,1.2,2.9,7,17,41,100 \mu \mathrm{m}$; c) The activation energy of drain current shows similar behaviour to transconductance, with corresponding kinks at the same gate voltages. Top to bottom $S=0.2,0.5,1.2,2.9,7,17,41$, $100 \mu \mathrm{m}$. For all panels $\phi_{\mathrm{B} 0}=0.5 \mathrm{eV}, \alpha=0$ and $\beta=1$. 


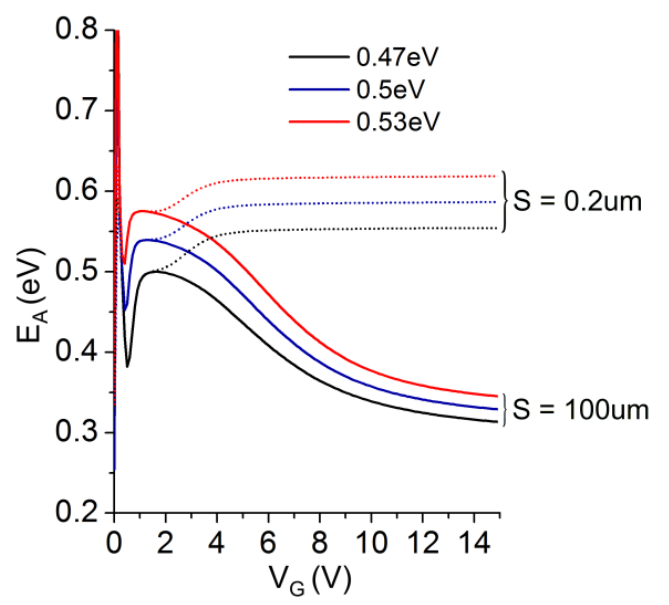

FIG. 3. Simulated activation energy vs. gate voltage plots for thee source barrier values and extreme source lengths $\left(\phi_{\mathrm{B} 0}=\right.$ $0.5 \mathrm{eV}, \alpha=0$ and $\beta=0)$; 
a)

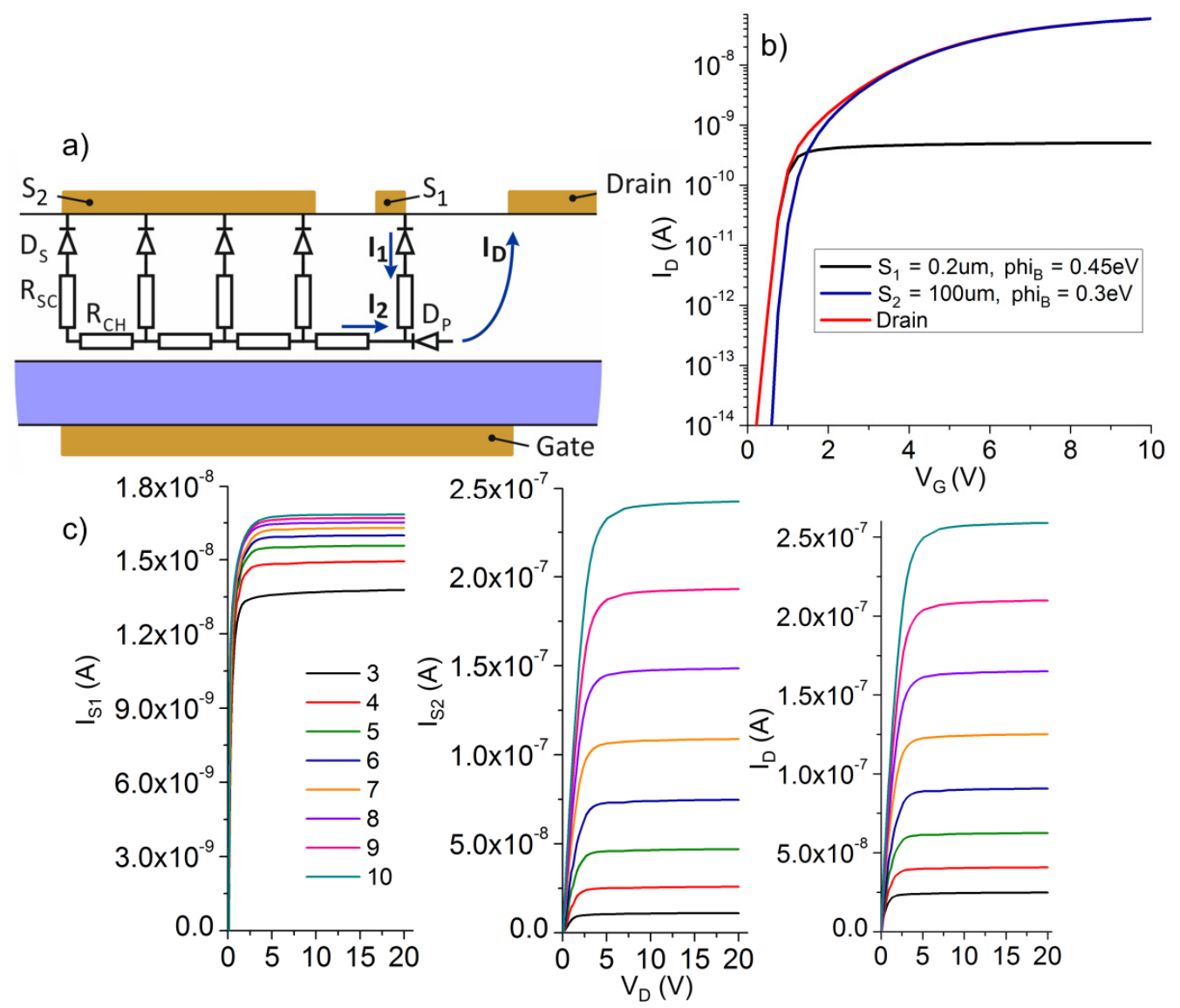

FIG. 4. a) schematic cross-section of the didactical structure, showing the location and relative size of the two source electrodes; b) simulated transfer characteristics for the structure in a) showing the contribution of the currents injected by electrodes $S_{1}$ and $S_{2}$ to the total drain current, for $V_{D}=5 \mathrm{~V} ; \mathrm{c}$ ) output characteristics of same showing the saturation profile and the relative magnitude of the $S_{1}, S_{2}$, and drain current, for barrier lowering coefficients $\alpha=\beta=0$ and eight gate bias values. 

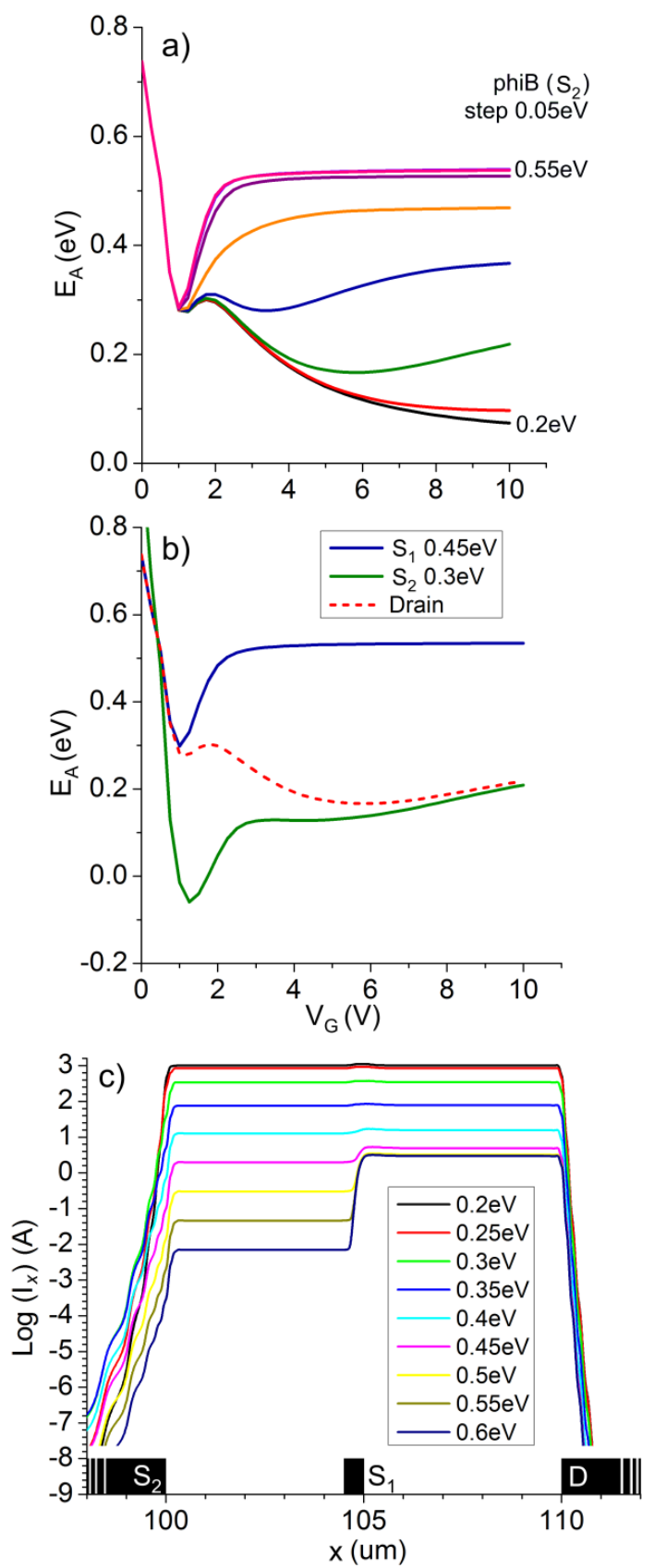

FIG. 5. Simulated behavior of SGT with split source. a) Activation energy of drain current vs gate voltage for different values of potential barrier at electrode $S_{2}$, while keeping $\phi_{\mathrm{B} 0}\left(S_{1}\right)=0.45 \mathrm{eV}$ constant; b) Activation energies of the currents at electrodes $S_{1}, S_{2}$ and $D$, showing that the drain current activation energy approaches that of the current of $S_{2}$ when $S_{2}$ contributes the largest proportion of drain current; c) Cutline at the insulator-semiconductor interface showing total current in the X direction vs. position, for varying $\phi_{\mathrm{B} 0}\left(S_{2}\right)$ and constant $\phi_{\mathrm{B} 0}\left(S_{1}\right)=0.45 \mathrm{eV}$, illustrating the contribution of $S_{1}$ and $S_{2}$. $\alpha=\beta$ $=0$ throughout. $S_{2}=95 \mu \mathrm{m}$. 